\title{
Research and Scholarly Activity in US Anesthesiology Residencies: A Survey of Program Directors and Residents
}

\author{
Viviane G. Nasr, Iqbal Ahmed, Iwona Bonney, and Roman Schumann \\ Department of Anesthesiology, Tufts Medical Center, Tufts University School of Medicine, 800 Washington Street, P.O. Box 298, \\ Boston, MA 02111, USA \\ Correspondence should be addressed to Viviane G. Nasr, vnasr@tuftsmedicalcenter.org
}

Received 31 January 2012; Accepted 13 February 2012

Academic Editors: A. Mizutani and A. Wiebalck

Copyright ( 92012 Viviane G. Nasr et al. This is an open access article distributed under the Creative Commons Attribution License, which permits unrestricted use, distribution, and reproduction in any medium, provided the original work is properly cited.

\begin{abstract}
Background. Effective 2007, the ACGME required scholarly activity during residency. Although many programs have ongoing research, residents' involvement may be limited. This US anesthesiology residency survey assesses the current scholarly environment, research activity and program support during training. Methods. Following IRB approval, 131 US anesthesiology program directors were invited to participate in a web-based survey. Questions to directors and residents included program structure, research activity, funding and productivity. We categorized residencies threefold based on their size. Results are summarized descriptively. Results. The response rate was 31.3\% $(n=41)$ for program directors and $15.3 \%(n=185)$ for residents. Residents' responses mirrored those of program directors' regarding the presence of didactic curricula (51\% versus 51.9\%), research rotations (57\% versus $56.2 \%)$ and a project requirement (37\% versus $40 \%)$. Demands of residency (27.0\%) and early stage in training $(22.2 \%)$ were the main obstacles to research cited by trainees. Residents' financial support was available in $94.3 \%$ of programs. Medium and large programs had multiple funding sources (NIH, industrial and private). Conclusion. Programs are dedicated to incorporate research into their curriculum. Residents' financial support and mentorship are available, while research time is limited. Systematic improvements are needed to increase trainee research in US anesthesiology residencies.
\end{abstract}

\section{Introduction}

The Common Program Requirements (CPRs) developed by the Accreditation Council for Graduate Medical Education (ACGME) in the United States effective in July $2007 \mathrm{em}$ phasize research and scholarly activity for residents and faculty in all residency programs. Specifically the CPR states, "Faculty should encourage and support residents in scholarly activities" and "The curriculum must advance residents' knowledge of the basic principles of research, including how research is conducted, evaluated, explained to patients, and applied to patient care. Residents should participate in scholarly activity. The sponsoring institution and program should allocate adequate educational resources to facilitate resident involvement in scholarly activities" [1]. Residency review committees will then apply specialty specific stipulations. These requirements appear to be in line with the progress of many medical specialties including anesthesiology, whose continued development and improvement of its clinical practice is a direct result of academic efforts and research. However, as for the specialty of anesthesiology in the USA, there has been recent increased awareness regarding the limited research in this field and its impact on the specialty.

For example, in a keynote address Reves states, "Our research problem is not so much one of poor quality but rather of insufficient quantity." [2] Szokol et al. identified a decline of American authorship in Anesthesiology and Anesthesia \& Analgesia from $80 \%$ to $44 \%$ between 1980 and 2000 [3]. In 1989 the duration of the residency in anesthesiology was increased to 4 years. Following this increase, the number of one-year research fellows declined from 31\% $(n=19$ out of 62 fellows who pursued 12 months subspecialty training) of total fellows in 1989 to $2 \%(n=7$ out of 383 fellows who pursued 12 months subspecialty training) in 2000 [4]. A sense within the anesthesiology community that 
TABLE 1: Residency research infrastructure: program director perspective.

\begin{tabular}{|c|c|c|c|c|}
\hline & $\begin{array}{l}\text { Small programs } \\
\quad(n=20)\end{array}$ & $\begin{array}{l}\text { Midsize programs } \\
\quad(n=6)\end{array}$ & $\begin{array}{l}\text { Large programs } \\
\qquad(n=9)\end{array}$ & $\begin{array}{c}\text { All } \\
(n=35)\end{array}$ \\
\hline Didactic research program during residency & $65 \%(13)$ & $66 \%(4)$ & $11 \%(1)$ & $51 \%(18)$ \\
\hline Dedicated research rotation during residency & $40 \%(8)$ & $100 \%(6)$ & $67 \%(6)$ & $57 \%(20)$ \\
\hline Mandatory research project during residency & $35 \%(7)$ & $66 \%(4)$ & $22 \%(2)$ & $37 \%(13)$ \\
\hline Educational allowance & $80 \%(16)$ & $83 \%(5)$ & $56 \%(5)$ & $74 \%(26)$ \\
\hline Stipend for meeting presentation & $100 \%(20)$ & $100 \%(6)$ & $78 \%(7)$ & $94 \%(33)$ \\
\hline Dedicated residency research mentor & $80 \%(16)$ & $83 \%(5)$ & $78 \%(7)$ & $80 \%(28)$ \\
\hline Residents assigned to research mentor & $50 \%(10)$ & $83 \%(5)$ & $56 \%(5)$ & $57 \%(20)$ \\
\hline Nonclinical time for research & $40 \%(8)$ & $100 \%(6)$ & $44 \%(4)$ & $51 \%(18)$ \\
\hline Nonclinical time not specifically for research & $45 \%(9)$ & $67 \%(4)$ & $33 \%(3)$ & $46 \%(16)$ \\
\hline
\end{tabular}

this trend may have reversed since could not be substantiated for a lack of more recent statistics. USA anesthesiology departments receive only $1 \%$ of National Institutes of Health (NIH) funding even though anesthesiologists represent 6\% of the physician workforce [5]. When comparing NIH award amounts per faculty member, in the USA anesthesiology is second to last ahead of family medicine. When looking at our specialty's leaders, anesthesiology department chairs average fewer publications and $\mathrm{NIH}$ grants compared to their surgical counterparts, 50 versus 133 publications, and $30 \%$ versus $62 \%$ personal NIH grants, respectively [6]. The reasons for these numbers are multifactorial and complex, but the new ACGME focus on research for residents and appropriately also for faculty may be beneficial for this specialty.

We conducted a survey among US anesthesiology residency directors and their residents to determine and better understand compliance with the ACGME requirements for scholarly activity and elucidate attitudes, perceptions, and possible obstacles including funding, mentorship, and time towards research during anesthesiology residency.

\section{Methods}

Following Institutional Review Board (IRB) approval, an introductory letter explaining the web-based survey and a questionnaire website was mailed to all program directors at 131 accredited anesthesiology programs in the United States in October 2009. A reminder email and the survey link were sent a total of 3 times over a 3-month period between November 2009 and January 2010. The survey to program directors included 21 questions (Appendix A) addressing the structure of the residency program, research activity, funding, and productivity. Program directors were asked in the cover letter to forward to their residents a separate electronic survey, the content of which was included for their review and approval (Appendix B). This anonymous voluntary survey for anesthesiology residents consisted of 28 slightly different questions to assess residents' research rotations, productivity, possibility of presentation at meetings, and satisfaction with their residency.

Responses returned via the survey site were entered into an excel database, and descriptive statistics including percentages were applied. Further statistical analysis depended on the response rate achieved.

\section{Results}

The overall response rate of program directors was $31.3 \%$ ( $n=41$ of 131 programs). Thirty-five programs completed the survey $(26.7 \%)$ and 6 program directors indicated their refusal to participate. No response at all was noted from the remaining 90 anesthesiology residency program directors.

We stratified the residencies based on the number of residents into small $(\leq 30, n=20)$, medium $(31-45, n=6)$, and large programs $(\geq 46, n=9)$. Within these categories, the program response rate was as follows: small programs $33.3 \%(n=20 / 60)$, medium programs $18.75 \%(n=$ $6 / 32)$, and large programs $23.1 \%(n=9 / 39)$. Half of the residencies $(51 \%)$ had a didactic curriculum specific for research education, especially the small and medium sized (65\% and 66\%, resp.) compared to larger programs (11\%). A dedicated research rotation existed in $57 \%$ of the residencies and $48.6 \%$ of residents have their research rotation during the CAIII year (Figure 1). All medium-sized programs have a research rotation during residency training. Anesthesiology residencies that responded had at least one or more residents actively involved in research at the time of the survey.

Departmental support for residents' research financially was available in $94.3 \%$ of program and for research mentorship in $80 \%$ of programs. Scheduled nonclinical time dedicated for research was made available in $51 \%$ of residencies. Results for the residency infrastructure-didactics, mentorship, financial support, and nonclinical time-are detailed in Table 1.

Research funding obtained by departments was classified into NIH, industrial, and private grants. All medium and large-sized programs had multiple sources of research funding, compared to $60 \%$ of small programs, $15 \%$ of which had no research funding at all (Table 2).

Residents' responses were dependant on whether or not they received the survey from their respective program directors, and whether or not they subsequently would choose to participate in this anonymous, voluntary study. The number of US anesthesiology residents at the time of 


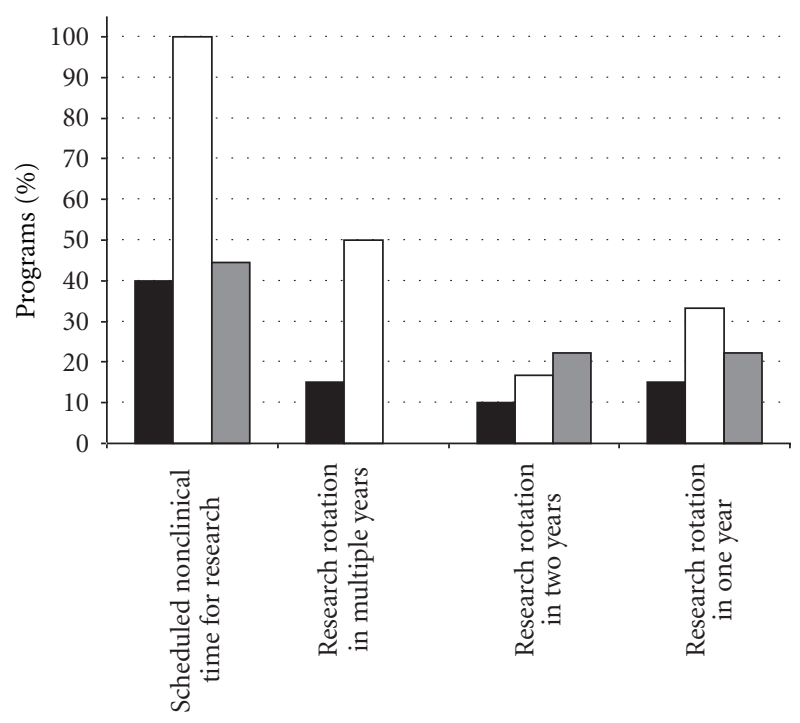

$$
\begin{aligned}
& \text { Programs: } \\
& \quad \text { Small ( } \leq 30 \text { residents) } \\
& \square \text { Medium ( } 31-45 \text { residents) } \\
& \square \text { Large ( }>45 \text { residents) }
\end{aligned}
$$

Figure 1: Research time based on the Clinical Anesthesia year and program size.

Table 2: Research Funding.

\begin{tabular}{lcccc}
\hline Programs & $\begin{array}{c}\text { NIH } \\
\text { grants }\end{array}$ & $\begin{array}{c}\text { Multiple } \\
\text { Funding* }\end{array}$ & $\begin{array}{c}\text { Single } \\
\text { Funding }\end{array}$ & $\begin{array}{c}\text { No } \\
\text { funds }\end{array}$ \\
\hline Small $(n=20)$ & $20 \%(4)$ & $60 \%(12)$ & $25 \%(5)$ & $15 \%(3)$ \\
Mid-size $(n=6)$ & $100 \%(6)$ & $100 \%(6)$ & 0 & 0 \\
Large $(n=9)$ & $100 \%(9)$ & $100 \%(9)$ & 0 & 0 \\
\hline
\end{tabular}

${ }^{*}$ Multiple funding sources include two or more sources of funding including NIH funding, industrial funding, private grants, and departmental funds.

tSingle funding source means that only one source is available for funds among the afore mentioned sources.

launching the survey during the year 2009 was 4720 including CAI to CAIII residents [7]. One-hundred eighty-five residents responded to the survey, reflecting a $4 \%$ national response rate. However, assuming that only responding program directors forwarded the survey to their residents, the resident survey population numbered 1212 trainees. Based on this statistic $15.3 \%$ (185/1212) of the resident study population was captured. The distribution of residents who completed the survey by the stage of their training was as follows: CAI $=37.3 \%(69 / 185)$, CAII $=34.0 \%(63 / 185)$, and CAIII $=28.7 \%(53 / 185)$. Responses of the residents are presented in Tables 3 and 4 . They were comparable to the program directors regarding didactic efforts for research (51\% versus $51.9 \%)$ and dedicated research rotations $(57 \%$ versus $56.2 \%$ ) (Table 3 ). Forty percent of residents and 37\% of residency program directors consider the accomplishment of or involvement in a research project mandatory. Residents considered either the CAI or II year the best time during residency to get involved in research, when the current situation suggests that residents are mostly involved only at the CAIII level. Table 5 summarizes the most frequent reasons for residents who have not started a research project. The demands of residency $(27.0 \%)$ and early stage in training with limited awareness for research $(22.2 \%)$ were the most common.

\section{Discussion}

Recently, US anesthesiology teaching departments have become more aware of the need to include research into the residency curriculum. One reason is a general realization of the limited research performance within the field of anesthesiology compared to other medical specialties. Another important factor was the emphasis placed on scholarly activity and research for residents by the ACGME requirements introduced in 2007. Residency Review Committees (RRCs) are now critically appraising trainee's understanding of research as it pertains to patient care and their involvement in scholarly or research activity. Indeed, each resident should complete an academic assignment, and the sponsoring institution is accountable to provide an appropriate scholarly environment and its attendant resources. Likewise, faculty scholarly and research performance is more stringently considered in each program. Effective July 1, 2008, the ACGME expects that the outcomes of resident investigations will be "suitable for presentation at local, regional, or national scientific meetings and that many will result in peer-reviewed abstracts or manuscripts". Therefore, an important mission of a residency is to educate its residents to appreciate the necessary work, skill set, and time expended by clinician researchers to do academic work and to teach critical evaluation of the scientific literature.

The results of our study provide a limited understanding of the current status of anesthesiology residents' research involvement from a program director's as well as resident's perspective, largely because of the low response rate. Just half of the surveyed programs had a didactic program and a dedicated research rotation indicating these types of efforts to fulfill the ACGME requirements.

Perceived obstacles for anesthesiology trainee research participation are similar to those identified by internal medicine residents and include time constraints and a lack of interest by recruited residents as well as by the larger faculty [8]. A recent study among Canadian anesthesiology residents concluded the same, namely, insufficient protected research time, interest, and curriculum deficiency regarding research that prevented their involvement [9]. Illustrating current production pressure in anesthesiology training programs, one resident in our study commented: "Despite many efforts, expressed interest, and strong desire, the financial pressure to do clinical work and "produce" makes it hard for residents to do research". Encouragingly, in our study, $44 \%$ of the programs provided some dedicated nonclinical research time for their residents.

The anesthesiology candidates attracted to this field as medical students have rated "time off" among the top five reasons for their specialty choice as shown by Wass et al. [10]. Fleisher and Eckenhoff acknowledge the need for an 
TABLE 3: Residency research infrastructure: resident perspective.

\begin{tabular}{|c|c|c|c|}
\hline & Yes & No & Not applicable \\
\hline Didactic research program during residency & $51.9 \%(96)$ & $48.1 \%(89)$ & \\
\hline Dedicated research rotation during residency & $56.2 \%(104)$ & $43.8 \%(81)$ & \\
\hline Mandatory research project during residency & $40.0 \%(74)$ & $58.4 \%(108)$ & $1.6 \%(3)$ \\
\hline Is the research rotation mentored & $56.7 \%(105)$ & $6.5 \%(12)$ & $36.8 \%(68)$ \\
\hline Adequate mentoring for the research project & $51.4 \%(95)$ & $9.2 \%(17)$ & $39.4 \%(73)$ \\
\hline Project is worth the time and commitment & $45.9 \%(85)$ & $8.6 \%(16)$ & $45.5 \%(84)$ \\
\hline Recommend involvement in projects to fellow residents & $71.4 \%(132)$ & $9.7 \%(18)$ & $18.9 \%(35)$ \\
\hline Research involvement and residency satisfaction & $38.9 \%(72)$ & $16.2 \%(30)$ & $44.9 \%(83)$ \\
\hline
\end{tabular}

TABLE 4: Timing of research during residency.

(a) Timing of research rotation

\begin{tabular}{lccccc}
\hline Residents' year & $\begin{array}{c}\text { One } \\
\text { year }\end{array}$ & $\begin{array}{c}\text { Two } \\
\text { years }\end{array}$ & $\begin{array}{c}\text { Three } \\
\text { years }\end{array}$ & $\begin{array}{c}\text { Four } \\
\text { years }\end{array}$ & $\begin{array}{c}\text { Not } \\
\text { applicable }\end{array}$ \\
\hline CAI $(n=69)$ & 21 & 9 & 4 & 2 & 33 \\
CAII $(n=63)$ & 22 & 11 & 3 & 0 & 27 \\
CAIII $(n=53)$ & 21 & 9 & 3 & 2 & 18 \\
\hline \multirow{2}{*}{ Total } & 64 & 29 & 10 & 4 & 78 \\
& $(34.6 \%)$ & $(15.6 \%)$ & $(5.4 \%)$ & $(2.2 \%)$ & $(42.2 \%)$ \\
\hline
\end{tabular}

(b) The best time to get involved in a project during residency per residents' opinion

\begin{tabular}{lccccc}
\hline & CA1 & CA2 & CA3 & CA4 & $\begin{array}{c}\text { No specific } \\
\text { period }\end{array}$ \\
\hline CAI $(n=69)$ & 23 & 23 & 9 & 1 & 13 \\
CAII $(n=63)$ & 19 & 22 & 8 & 0 & 14 \\
CAIII $(n=53)$ & 21 & 20 & 7 & 1 & 4 \\
\hline Total & 63 & 65 & 24 & 2 & 31 \\
& $(34.1 \%)$ & $(35.1 \%)$ & $(13.0 \%)$ & $(1.1 \%)$ & $(16.7 \%)$ \\
\hline
\end{tabular}

TABLE 5: Reasons given by residents not involved in research.

\begin{tabular}{lc}
\hline Reasons why you have not done a research project yet & Response \\
\hline I am in the stage in life where I cannot make research & $27.0 \%(50)$ \\
a priority in addition to surviving the residency & \\
I haven't thought about this, I am only CAI & $22.2 \%(41)$ \\
It is too much work & $13.5 \%(25)$ \\
I will not be given the time that it will take & $12.4 \%(23)$ \\
I am not interested & $10.8 \%(20)$ \\
It is not required in my residency & $10.8 \%(20)$ \\
It really is not part of the "culture" in my residency & $9.2 \%(17)$ \\
\hline
\end{tabular}

image hange of this specialty, which has to begin in medical school [11]. Medical students are attracted to the specialty of anesthesiology by lifestyle [6], a relatively short residency [12], its procedural orientation, the practice of physiology and pharmacology, controllable work hours including part time practice, instant gratification, and the perception of ample time off $[10,13]$. Research per se, but particularly in addition to clinical practice, will frequently require strong individual motivation in addition to extended work hours beyond time spent in the clinical environment. This notion will clash with the expectations of many who currently choose anesthesiology as a profession. Wass et al. [10] demonstrated the decline in research interest over the past decade and the opportunity to conduct research was less important as a factor in choosing an anesthesiology training program (63\% in $1995-1996$ versus $27 \%$ in $2000-2001)$. In 2000-2001, private anesthesiology practice became more attractive as its earning potential had increased to be up to $30 \%$ above the level of academic practice [10].

The resident's view of anesthesiology is formed in myriad ways: mentorship, motivated faculty, and departmental opportunities to learn are some. To foster a culture of education and learning that includes research requires supportive personnel including but not limited to mentorship. Despite availability of dedicated research mentorship in $80 \%$ of the residencies according to program directors, only $56.7 \%$ of the residents reported mentored research rotation and only $51.4 \%$ of residents involved in research perceived mentoring as adequate. However, research pursuit is not solely a mentor's responsibility. It is rather the entire training environment. Access to time, mentorship, internal peer review, acquisition of medical writing skills, and administrative and statistician support are all essential for meaningful and successful research [14].

These desirable residency attributes in favor of research do come at an expense, which is systematically limiting factor for many US programs. Currently the distribution of NIH funding within anesthesiology is out of balance with $40 \%$ $(n=51)$ of 128 accredited anesthesiology departments receiving $\mathrm{NIH}$ grants while 19\% $(n=10 / 51)$ of those departments receive $48 \%$, and $38 \%(n=20 / 51)$ receive $77 \%$ of the total grants $[5,15]$. Whether or not, and if so, how to alleviate this uneven distribution is not clear. In our survey, funding for research was more consistent in midsize and large programs, all of which had multiple funding sources. This type of funding was clearly more difficult for smaller programs. Interestingly, individual resident's financial support for research meeting related expenses was more favorable in small compared to large residencies.

It would be desirable to compare our results to the current status of anesthesiology research during training of different countries and determine attributes most favorable 
to fulfill this mission. It may then be useful to entertain a comparison to different medical specialties, to uncover possible specialty specific obstacles.

Our study has several limitations. Survey fatigue may have contributed to our relatively low response rate $(31.3 \%$ of program directors and $4 \%$ of residents nationally). Assuming strong responder bias for our survey topic, it may be possible that the large proportion of programs not responding may have done so because of their own shortcomings regarding residency research. This would indicate that much work remains to be done to meet the intent of the ACGME scholarly activity requirements for residents in many US programs. Although the limited response to the survey does not allow generalization of our findings to all US residencies, the fact that 90 program directors chose not to respond at all despite multiple reminders and 6 directors directly declined participation on this topic can be interpreted as a troublesome result in and of itself. Nevertheless, even surmising a positive responder bias in this study, programs less prepared for trainee research may be able to gain insights from our select responders, as they try to comply with US ACGME requirements and the need to improve anesthesiology research in general.

We elected to not approach residents independently of their program directors, by coupling their engagement in the survey via the program directors assistance. Our intent was to leave this step at the program directors discretion, having fully disclosed the residents survey questions to this gate keeper. While offering maximum transparency and control of the study to program directors, this approach likely prevented a much higher capture of residents' responses.

Finally assessment of residency faculty and barriers to the establishment of a culture of research in anesthesiology departments were not at all assessed in this study but may also be needed to derive comprehensive strategies for improvement.

Some developments in recent years point to some progress in favor of anesthesiology research independently of ACGME mandates. The American Society of Anesthesiologists (ASA) and several additional national and international anesthesiology societies have recognized the value of research for this field and added research to their programs in recent years. Additionally, many of these professional organizations have created their own research agendas and established annual grants for junior faculty and residents alike. A general trend to encourage translational research across specialties including the basic sciences is potentially expanding the opportunities for research participation of our specialty. Some residencies have implemented research tracks such as the Duke Academic Career Enrichment Scholars program (http://anesthesia.mc.duke.edu/modules/ anes_resdcy/index.php?id=9) and the Stanford Fellowship in Anesthesia Research and Medicine program (http://med .stanford.edu/anesthesia/education/residentresearch.html). The American Board of Anesthesiology (ABA) and the ACGME supports research during residency allowing approximately $25 \%$ of a 3 - or 4 -year training program and more than $35 \%$ of a 5 -year program, engaged in scholarly activities. The ABA's Credentials Committee will consider requests for individual residents for additional research during the training. These changes represent the commitment of the anesthesiology profession to reemphasize research for the specialty and make it happen.

In conclusion, quoting Miller “... research is vital to the future of anesthesiology as a profession ...", it is timely that the ACGME requirements highlight the same point for all medical specialties [16]. Results of our study show awareness and a dedicated effort in the responding programs to incorporate research into the residency curriculum using didactics and research rotations. Financial support and mentorship are available in most programs, while dedicated research time is limited. The infrastructure for research appears to be particularly favorable in midsize programs. A systematic approach to solve insufficient research activity in anesthesiology residencies requires a complex, integrated strategy that includes medical schools, residencies, residency regulatory agencies, and adequate funding sources in a climate that promotes the importance of research for medicine at the student and resident level. Recruiting graduated residents with research interest and retaining junior faculty in anesthesiology programs, though difficult, is a must. Nationally and internationally, anesthesiology's research may greatly benefit from education in research skills into medical school curricula complementing the traditional teaching of medicine.

\section{Appendix}

\section{A. Survey Addressed to Program Directors}

(1) How many resident positions do you have in your program?

$$
\begin{aligned}
& \mathrm{CA} \mathrm{I}= \\
& \mathrm{CA} \mathrm{II}= \\
& \mathrm{CA} \mathrm{III}= \\
& \mathrm{CAIV} \text { (fellows) = }
\end{aligned}
$$

(2) Does your residency include a didactic program (i.e. lecture series) for research?

(1) Yes

(2) No

(3) Do your residents have a research rotation?

CA I:

(1) Yes

(2) No

CA II:

(1) Yes

(2) $\mathrm{No}$

CA III:

(1) Yes

(2) $\mathrm{No}$ 
(4) Is research mandatory for the residents in your program?

(1) Yes

(2) No

(5) How many residents are currently involved in a research project?

$$
\begin{aligned}
& \mathrm{CA} \mathrm{I}= \\
& \mathrm{CA} \mathrm{II}= \\
& \text { CA III= }
\end{aligned}
$$

(6) How many research projects are currently ongoing at your department?

(7) How many of these projects include residents?

(8) How many publications in peer-reviewed journals did your department have during the past academic year?

(9) How many of these publications included a resident as author/coauthor?

(10) How many abstracts/posters/oral communications did your department have during the past academic year?

(11) How many residents were authors/coauthors on

$$
\begin{aligned}
& \text { Abstracts } \\
& \text { Posters } \\
& \text { Oral Communications }
\end{aligned}
$$

(12) Does each resident have an educational allowance?
(1) Yes
(2) No

(13) Do residents receive separate financial support to attend scientific anesthesia meetings when they present an abstract?
(1) Yes
(2) No
Please describe:

(14) Does the department have a dedicated research mentor for residents?
(1) Yes
(2) No

(15) Is each resident assigned a mentor for research?
(1) Yes

(2) $\mathrm{No}$

(16) Do residents have scheduled nonclinical time assigned for research?

CA I:
(1) Yes
(2) No

CA II:
(1) Yes
(2) No

CA III:

(1) Yes

(2) No

(17) Do residents have nonclinical time not specifically assigned to research?

\section{CA I:}

(1) Yes

(2) No

CA II:

(1) Yes

(2) No

CA III:

(1) Yes

(2) No

(18) Does your department have NIH-funded research?
(1) Yes
(2) No

(19) Does your department have industry-funded research?
(1) Yes
(2) No

(20) Does your department have private grant-funded research?
(1) Yes
(2) $\mathrm{No}$

(21) Does your department allocate funds for research that are not from grants?

(1) Yes

(2) No 


\section{B. Survey Addressed to Residents}

(1) I am a
(1) CA I
(2) CA II
(3) CA III
(4) CA IV (fellowship)

Please name the fellowship subspecialty.

(2) Please rate your satisfaction overall with your residency program:
(1) Not satisfied
(2) Somewhat satisfied
(3) Satisfied
(4) Very satisfied

(3) My residency has a specific research curriculum (lecture series, mentorship, etc.):
(1) Yes
(2) No

(4) My residency has a specific research rotation with nonclinical time:
(1) Yes
(2) No

(5) A research rotation occurs during (check all that applies)

$$
\begin{aligned}
& \text { CA1 } \\
& \text { CA2 } \\
& \text { CA3 } \\
& \text { CA4 (fellowship) }
\end{aligned}
$$

(6) Is the research rotation mentored?
(1) Yes
(2) No
(3) Not applicable

(7) Information about ongoing research and opportunities for residents in my department is easy to obtain and very transparent:
(1) Yes
(2) No
(3) Not applicable

(8) Research participation or a project is mandatory in my program:
(1) Yes
(2) No
(3) Not applicable

(9) Are you involved in research/abstract/case report projects intended for presentation or publication during this residency program?
(1) Currently involved
(2) Completed
(3) Not involved
(4) Going to be involved

(10) Did you receive adequate mentoring for your project?
(1) Yes
(2) No
(3) Not applicable

(11) Have you authored or coauthored a research related publication (original article/case report/letter/ abstract/review, etc.) during this residency/fellowship?
(1) Yes
(2) No
(3) Not Applicable

(12) Did you present at any extradepartmental meeting/ conference during this residency?
(1) Yes
(2) No
(3) Not applicable

(13) Was the presentation at an anesthesia meeting?
(1) Yes
(2) No
(3) Not applicable

(14) Did you have adequate time for your project?

(1) Yes

(2) No

(3) Not applicable

(15) Did your research involvement increase your satisfaction with the residency?
(1) Yes
(2) No
(3) Not applicable

(16) Did you feel your project was worth the time and commitment that you invested?
(1) Yes
(2) No
(3) Not applicable

(17) How many academic projects were you involved in during this residency?

Not applicable $\quad \begin{array}{lll}1 & 2 & >2\end{array}$


(18) Was this research funded by sources outside the department (grant, industry, etc.)?
(1) Yes
(2) No
(3) Don't know
(4) Not applicable

(19) Was the funding source?
(1) Private
(2) Federal
(3) Industry
(4) Do not Know
(5) Not applicable

(20) In your opinion, when is the best time to get involved in a project during residency?
(1) CA I
(2) CA II
(3) CA III
(4) CA IV (fellowship)
(5) No specific Period

(21) Would you recommend doing a project to your fellow residents?
(1) Yes
(2) No
(3) Not applicable

(22) If you are not involved in research, do you believe that such involvement would improve your satisfaction with the residency?
(1) Yes
(2) No
(3) $\mathrm{N} / \mathrm{A}$

(23) If you have NOT done a research project yet, check all the reasons that apply:

(1) I have not thought about this; I am only a CA I

(2) I am not interested

(3) It is too much work

(4) I will not be given the time that it will take

(5) It is not valued as an effort in my Department

(6) We do not have staff that will mentor and guide me

(7) I am in a stage in life where I cannot make research a priority in addition to surviving the residency

(8) It is not important for the specialty of anesthesiology

(9) I do not have any ideas, and I do not know whom to ask
(10) It really is not part of the "culture" in my residency

(11) It is not required in my residency

(12) My residency does not have a didactic research program for residents (i.e. lecture series, research rotation)

(13) Other-please explain:

(24) Is this your first postgraduate training?
(1) Yes
(2) No

The following part will continue if the answer to question 24 is yes:

(25) Were you involved in research in your previous training?
(1) Yes
(2) No
(3) Not applicable

(26) Have you authored or coauthored a research related publication (original article/case report/letter/ abstract/review, etc.) prior to this residency?
(1) Yes
(2) No
(3) Not applicable

(27) Did you present at any meeting/conference prior to this residency?

(1) Yes

(2) $\mathrm{No}$

(3) Not applicable

(28) Was the presentation at an anesthesia meeting?
(1) Yes
(2) No
(3) Not applicable

\section{Acknowledgment}

This paper was funded by the Department of Anesthesiology.

\section{References}

[1] Accreditation Council for Graduate Medical Education on Common Program Requirements, 2007, http://www.acgme .org/acWebsite/dutyHours/dh_dutyhoursCommonPR07012007.pdf.

[2] J. G. Reves, "We are what we make: transforming research in anesthesiology - the 45th Rovenstine lecture," Anesthesiology, vol. 106, no. 4, pp. 826-835, 2007. 
[3] J. W. Szokol, G. S. Murphy, M. J. Avram, M. Nitsun, T. M. Wynnychenko, and J. S. Vender, "Declining proportion of publications by American authors in major anesthesiology journals," Anesthesia \& Analgesia, vol. 96, no. 2, pp. 513-517, 2003.

[4] J. E. Havidich, G. R. Haynes, and J. G. Reves, "The effect of lengthening anesthesiology residency on subspecialty education," Anesthesia \& Analgesia, vol. 99, no. 3, pp. 844-856, 2004.

[5] D. A. Schwinn and J. R. Balser, "Anesthesiology physician scientists in academic medicine: a wake-up call," Anesthesiology, vol. 104, no. 1, pp. 170-178, 2006.

[6] D. J. Culley, G. Crosby, Z. Xie, C. A. Vacanti, R. J. Kitz, and W. M. Zapol, "Career National Institutes of Health funding and scholarship of chairpersons of academic departments of anesthesiology and surgery," Anesthesiology, vol. 106, no. 4, pp. 836-842, 2007.

[7] A. Schubert, "2009 anesthesiology resident class sizes and graduates," American Society of Anesthesiology, vol. 74, no. 2, pp. 34-38, 2010.

[8] S. Gill, A. Levin, O. Djurdjev, and E. M. Yoshida, "Obstacles to residents' conducting research and predictors of publication," Academic Medicine, vol. 76, no. 5, p. 477, 2001.

[9] L. C. Silcox, T. L. Ashbury, E. G. VanDenKerkhof, and B. Milne, "Residents' and program directors' attitudes toward research during anesthesiology training: a Canadian perspective," Anesthesia \& Analgesia, vol. 102, no. 3, pp. 859-864, 2006.

[10] C. T. Wass, T. R. Long, D. W. Randle, S. H. Rose, R. J. Faust, and P. A. Decker, "Recruitment of house staff into anesthesiology: a re-evaluation of factors responsible for house staff selecting anesthesiology as a career and individual training program," Journal of Clinical Anesthesia, vol. 15, no. 4, pp. 289-294, 2003.

[11] L. A. Fleisher and R. G. Eckenhoff, "Image not living up to goal," Anesthesiology, vol. 105, no. 3, pp. 626-627, 2006.

[12] S. Gelman, "Anesthesiologist scientist: endangered species," Anesthesiology, vol. 105, no. 3, pp. 624-625, 2006.

[13] L. J. Roberts and D. C. S. Khursandi, "Career choice influences in Australian anaesthetists," Anaesthesia and Intensive Care, vol. 30, no. 3, pp. 355-359, 2002.

[14] J. A. Campagna, "Academic anesthesia and M.D.-Ph.D.s," Anesthesiology, vol. 105, no. 3, pp. 627-628, 2006.

[15] P. R. Knight and D. C. Warltier, "Anesthesiology residency programs for physician scientists," Anesthesiology, vol. 104, no. 1, pp. 1-4, 2006.

[16] R. D. Miller, "The place of research and the role of academic anaesthetists in anaesthetic departments," Best Practice and Research: Clinical Anaesthesiology, vol. 16, no. 3, pp. 353-370, 2002. 


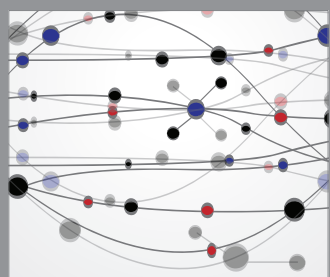

The Scientific World Journal
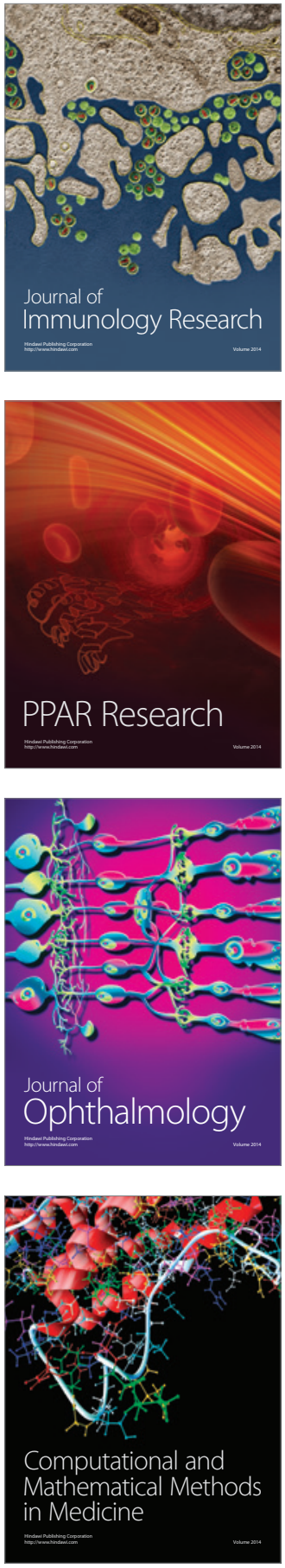

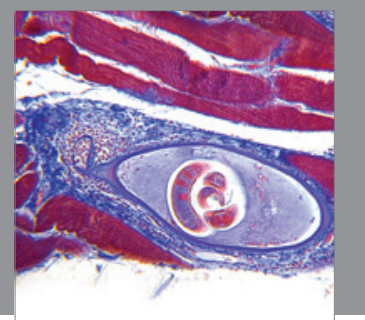

Gastroenterology

Research and Practice
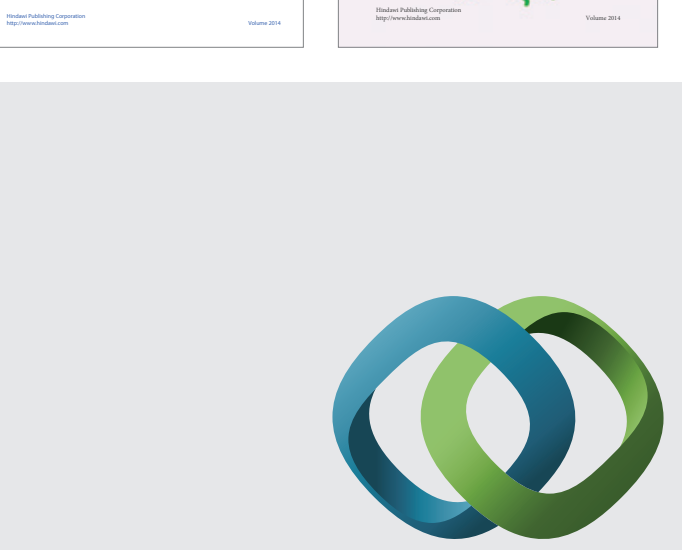

\section{Hindawi}

Submit your manuscripts at

http://www.hindawi.com
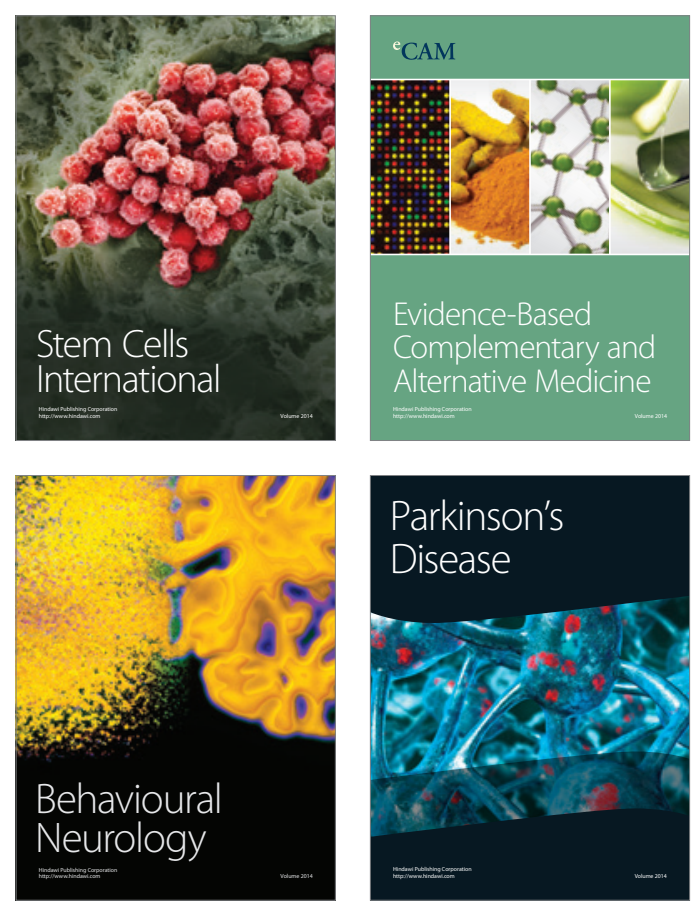

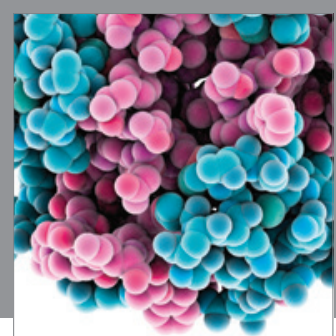

Journal of
Diabetes Research

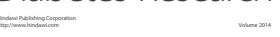

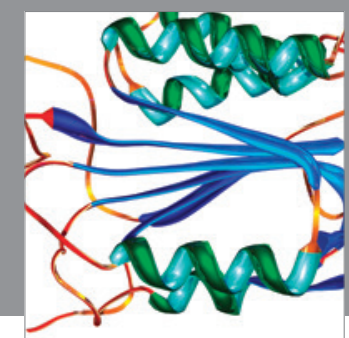

Disease Markers
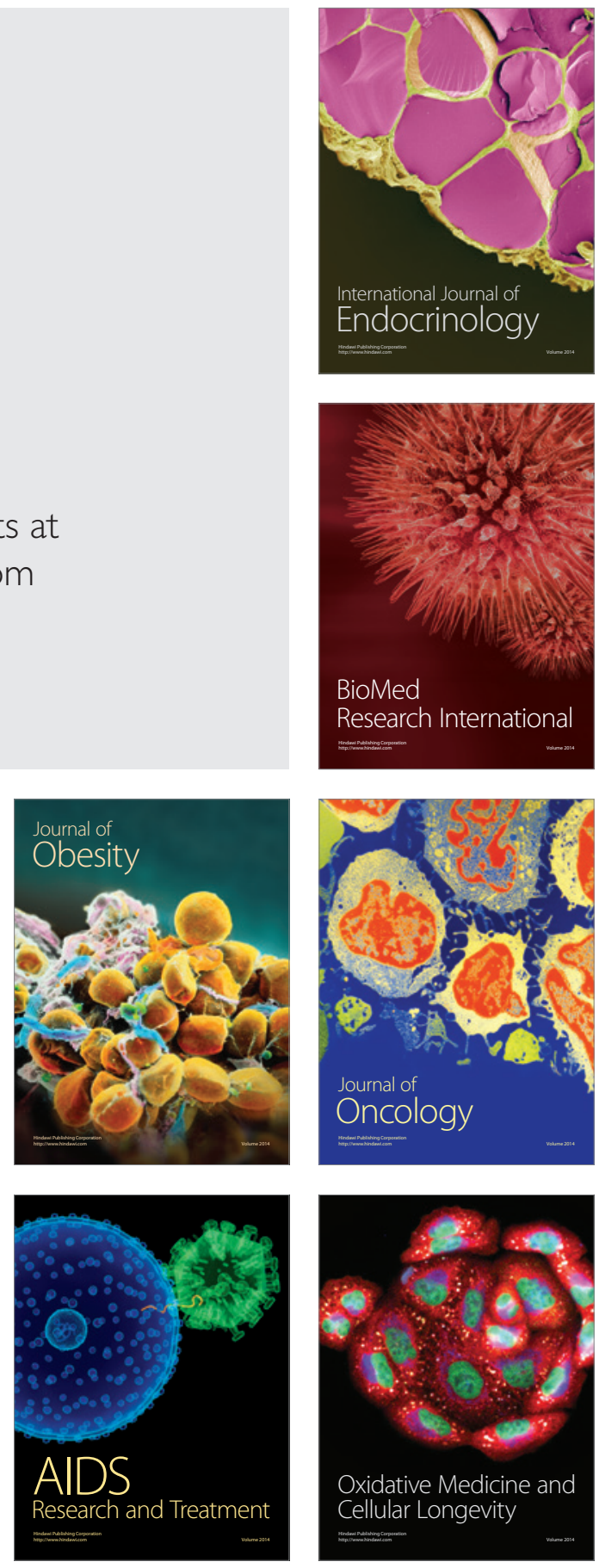